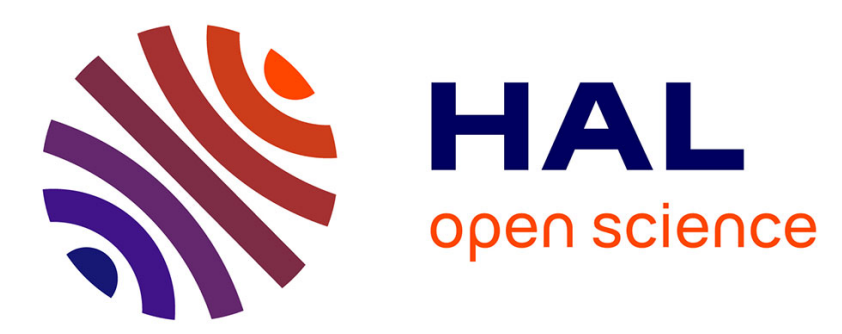

\title{
Scale invariance analysis for genetic networks applying homogeneity
}

\author{
Emmanuel Bernuau, Denis Efimov, Wilfrid Perruquetti
}

\section{To cite this version:}

Emmanuel Bernuau, Denis Efimov, Wilfrid Perruquetti. Scale invariance analysis for genetic networks applying homogeneity. Journal of Mathematical Biology, 2015, 72, pp.1-10. 10.1007/s00285-0150923-y . hal-01185119

\section{HAL Id: hal-01185119 \\ https://hal.inria.fr/hal-01185119}

Submitted on 19 Aug 2015

HAL is a multi-disciplinary open access archive for the deposit and dissemination of scientific research documents, whether they are published or not. The documents may come from teaching and research institutions in France or abroad, or from public or private research centers.
L'archive ouverte pluridisciplinaire HAL, est destinée au dépôt et à la diffusion de documents scientifiques de niveau recherche, publiés ou non, émanant des établissements d'enseignement et de recherche français ou étrangers, des laboratoires publics ou privés. 


\title{
Scale invariance analysis for genetic networks applying homogeneity
}

\section{Emmanuel Bernuau • Denis Efimov • Wilfrid Perruquetti}

Received: 17/04/2014 / Accepted: date

\begin{abstract}
Scalability is a property describing the change of the trajectory of a dynamical system under a scaling of the input stimulus and of the initial conditions. Particular cases of scalability include the scale invariance and fold change detection (when the scaling of the input does not influence the system output). In the present paper it is shown that homogeneous systems have this scalability property while locally homogeneous systems approximately possess this property. These facts are used for detecting scale invariance or approximate scalability (far from a steady state) in several biological systems. The results are illustrated by various regulatory networks.
\end{abstract}

\section{Introduction}

Many biological systems demonstrate an adaptation ability, i.e. they are capable of maintaining the levels of essential variables in the admissible bounds in the presence of wide deviations of the range of inputs [Alon, 2006]. In some cases they even demonstrate a stronger property called (approximate) fold change detection, when the output of the system becomes (almost) the same after the input scaling [Shoval et al., 2011, Skataric \& Sontag, 2012, Hamadeh et al., 2013]. In general, a system admits the scale invariance property if a change of stimulus from a background level $u_{0}$ to a new level $u$, or from $\lambda u_{0}$ to $\lambda u$ for some scalar $\lambda>0$, results in a time response of the system that is entirely scaled by a function of $\lambda$. To illustrate this property, consider the Repressilator model

Denis Efimov and Wilfrid Perruquetti

Non-A team at Inria Lille, Parc Scientifique de la Haute Borne, 40 avenue Halley, 59650 Villeneuve d'Ascq, France and CRIStAL (UMR-CNRS 9189), Ecole Centrale de Lille, BP 48, Cité Scientifique, 59651 Villeneuve-d'Ascq, France

Emmanuel Bernuau

Faculty of Engineering, University of Pisa, Largo Lucio Lazzarino 1, 56122 Pisa, Italy 
from [Elowitz \& Leibler, 2000] for $i=1,2,3$ :

$$
\begin{aligned}
\dot{m}_{i}(t) & =u(t)+\frac{\alpha}{1+p_{j_{i}}^{n}(t)}-m_{i}(t), \\
\dot{p}_{i}(t) & =\beta\left(m_{i}(t)-p_{i}(t)\right), \\
j & =\left[\begin{array}{lll}
3 & 1 & 2
\end{array}\right],
\end{aligned}
$$

where $m_{i}$ and $p_{i}$ represent the concentrations of mRNA and protein of the three components of Repressilator; the input $u(t)$ represents the number of protein copies per cell produced from a given promoter type during continuous growth in the presence of saturating amounts of repressor; the parameter $\alpha$ is the repressor-dependent transcription rate; the parameter $\beta$ is the protein synthesis and degradation rate. The repression of the transcription is described by a Hill kinetics with cooperativity degree $n$. Take $\alpha=10, \beta=0.25, n=2$,

$$
\begin{aligned}
& u(t)=\left\{\begin{array}{ll}
10 & \text { if } t \in[40,50] \\
0.01 & \text { otherwise }
\end{array},\right.
\end{aligned}
$$

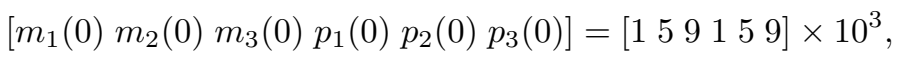

then the corresponding trajectories are shown in Fig. 1,a in logarithmic scale. Next, let us multiply the above initial conditions and $u(t)$ by $\lambda=10$, then the ratios of the resulting variables $m_{i}$ and $p_{i}$ with the corresponding variables obtained previously (given in Fig. 1,a) are presented in Fig. 1,b also in logarithmic scale. As we can see, these ratios equal "exactly" $\lambda$ if the amplitudes of $m_{i}$ and $p_{i}$ are high, which means that scaling the initial conditions and input $u(t)$ leads to a proportional scaling of the entire trajectories for high concentrations (not only at a steady state but also during transients). In other words, the system exemplifies scale invariance. Consequently, we see that big concentrations leakage scale is exactly transformed to concentration behavior.

Scale invariance has been studied for more than 20 years (see for instance [Gisiger, 2001] for a survey of this concept) and has proven to be useful in the process of designing models for biological systems. Indeed, if a real mechanism shows a kind of scale invariance (with respect to an input as defined beforehand, or simply by having an inner symmetry like fractals), its model should transcribe this fact by a mathematical property; conversely, if an accepted model has this mathematical property then the real process should have it too. Furthermore, assuming that a model is clearly stated and accepted, knowing precisely how this system will react to a given input change is extremely important for analyzing the system's behavior.

The scalability is particularly important for large-scale cascaded systems, like genetic regulatory networks, since it simplifies analysis of interconnection (let us recall that a cascaded system is a system where the state $x_{i}$ acts as the input of the subsystem given by the dynamics of $x_{i+1}$ ). For example, a (consecutive) connection of scale invariant systems leads to a scale invariant network, where the input, once applied to the first system, will be transformed to the output of the last one with the corresponding scale (or without it if all 

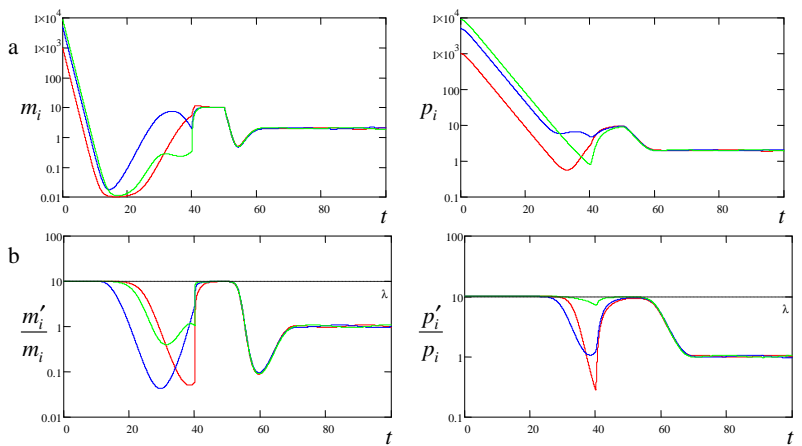

Fig. 1 Results of simulation for Repressilator: (a) the system trajectory; (b) the ratios between trajectory obtained for scaled by $\lambda$ input and initial conditions and the original one

cascaded systems have the fold change detection ability). For example, if we have a cascade of two dynamical systems, one transforms an input $u(t)$ to the state $x_{1}(t)$, and another the state $x_{1}(t)$ (as an input) to its own state $x_{2}(t)$, which serves as the output of the cascade. But in general it is hard to predict the output response $x_{2}(t)$ after a scaling of the input $u(t)$, since it depends on the dynamics of two systems. However, if both systems have the scale invariance or the fold change detection property, then the output will just be scaled accordingly. However, these approaches require us to have constructive ways to detect scale invariance in a mathematical model. Hence, constructive conditions to check the presence of scale invariance are demanded in systems biology for analysis and design.

The conditions of scale invariance obtained in [Shoval et al., 2011, Skataric \& Sontag, 2012, Hamadeh et al., 2013] are based on some kind of symmetry verification and linearization. It is shown that if a system is symmetric, then under some additional minor restrictions it has the scale invariance property. Homogeneity is a concept that has been introduced in order to study the influence of symmetry on the behavior (stability, robustness etc.) of solutions of nonlinear systems [Bacciotti \& Rosier, 2005, Kawski, 1995, Rosier, 1992]. Homogeneity is also a kind of symmetry. The verification of the presence of this type of symmetry is rather simple since it is based on arithmetic operations and/or algebraic tests.

The objective of this paper is to develop constructive strategies to verify the scale invariance property on biological systems models. In order to do that, we shall be using the homogeneity theory. We will first show that the homogeneity framework allows us to recast known results about scale invariance. We shall then establish that local homogeneity implies approximate scale invariance of the system. Finally, several examples demonstrating the efficiency of the homogeneity based methods for analyzing scale invariance are studied.

The outline of the paper is as follows. In Section 2 some preliminary results and definitions of homogeneity and scale invariance are presented. In Section 3 the link between homogeneity and scalability is established. The relation between approximate scalability and local homogeneity is investigated in Section 
4. After all mathematical developments, a discussion is given on application of these results for scale invariance detection in Section 5. Application to genetic cascades is considered in Section 6.

\section{Preliminaries}

\subsection{Notation}

In this paper, the following notation will be used.

- We denote $\mathbb{R}_{+}=\{x \in \mathbb{R}: x \geq 0\}$ and $\mathbb{R}_{+}^{*}=\mathbb{R}_{+} \backslash\{0\}$. If $E \subset \mathbb{R}$ we set $E_{+}=E \cap \mathbb{R}_{+}$and $E_{+}^{*}=E \cap \mathbb{R}_{+}^{*}$. We denote $\mathbb{N}=\mathbb{Z}_{+}$and $\mathbb{N}^{*}=\mathbb{Z}_{+} \cap \mathbb{R}_{+}^{*}$.

- If $n, p \in \mathbb{N}^{*}$, we denote $C^{0}\left(\mathbb{R}^{n}, \mathbb{R}^{p}\right)$ the set of continuous mapping from $\mathbb{R}^{n}$ to $\mathbb{R}^{p}$ and $\operatorname{Lip}\left(\mathbb{R}^{n}\right)$ the set of locally Lipschitz continuous vector fields on $\mathbb{R}^{n}$.

- Denote $\Psi$ the flow of a $F \in \operatorname{Lip}\left(\mathbb{R}^{n}\right)$, then $\Psi^{t}(x)$ represents the state of the trajectory of $F$ at time $t$ with the initial condition $x$, i.e. $\Psi^{0}(x)=x$. We denote $\frac{d}{d t} \Psi^{t}(x)$ the time derivative of this curve $\left(\frac{d}{d t} \Psi^{t}(x)=F\left(\Psi^{t}(x)\right)\right.$ by definition) and $d_{x} \Psi^{t}$ the differential at $x$ of the diffeomorphism $x \mapsto \Psi^{t}(x)$, $t$ being fixed.

- If $f$ is a differentiable vector field on $\mathbb{R}^{n}$, we denote Jac $f(x)$ the Jacobian matrix of $f$ taken at the point $x \in \mathbb{R}^{n}$.

- For two $C^{1}$ vector fields $f, g: \mathbb{R}^{n} \rightarrow \mathbb{R}^{n}$, denote their Lie brackets $[f, g]=$ $\frac{\partial g}{\partial x} f(x)-\frac{\partial f}{\partial x} g(x)$. For a $C^{1}$ vector field $f: \mathbb{R}^{n} \rightarrow \mathbb{R}^{n}$ and a $C^{1}$ function $V: \mathbb{R}^{n} \rightarrow \mathbb{R}$ the Lie derivative of $V$ along the vector field $f$ is defined as $\mathcal{L}_{f} V=\frac{\partial V}{\partial x} f(x)$.

- Given matrices $A_{k} \in \mathbb{R}^{n \times n}, 1 \leq k \leq K, K \in \mathbb{N}^{*}$, define the convex hull of these matrices

$$
\begin{gathered}
C o\left(A_{1}, \ldots, A_{K}\right)=\left\{A \in \mathbb{R}^{n \times n}: A=\sum_{k=1}^{K} \alpha_{k} A_{k},\right. \\
\left.\alpha_{k} \in[0,1], \sum_{k=1}^{K} \alpha_{k}=1\right\} .
\end{gathered}
$$

- For a symmetric matrix $A \in \mathbb{R}^{n \times n}$ denote $\lambda_{\max }(A)$ and $\lambda_{\min }(A)$ the biggest and the smallest eigenvalues, respectively.

- If $A$ and $B$ are two subsets of $\mathbb{R}^{n}$, we denote $A+B=\{a+b, a \in A, b \in B\}$.

- For a measurable function $u: \mathbb{R}_{+} \rightarrow \mathbb{R}^{m}$ define the norms $\|u\|_{\mathcal{L}^{1}[a, b]}=$ $\int_{a}^{b}|u(t)| d t$ and $\|u\|_{\mathcal{L}^{\infty}[a, b]}=\sup _{t \in[a, b]}|u(t)|$ for any $0 \leq a \leq b \leq+\infty$.

\subsection{Homogeneity concepts}

In order to use the symmetry that some systems present, the weighted homogeneity has been introduced [Zubov, 1958, Hermes, 1986]. 
Definition 1 For $\mathbf{r}=\left(r_{1}, \ldots, r_{n}\right)$ with $r_{i} \in \mathbb{R}_{+}^{*}$, a dilation associated to the generalized weight $\mathbf{r}$ is a group action: $\lambda \in \mathbb{R}_{+}^{*} \mapsto \Lambda_{\mathbf{r}}(\lambda)$ with $\Lambda_{\mathbf{r}}(\lambda): x \mapsto$ $\left(\lambda^{r_{1}} x_{1}, \ldots, \lambda^{r_{n}} x_{n}\right)$. The mapping $\Lambda_{\mathbf{r}}(\lambda)$ is often simply denoted by $\Lambda_{\mathbf{r}}$ (the $\lambda$ is omitted).

Definition 2 A function $V: \mathbb{R}^{n} \rightarrow \mathbb{R}$ (resp. a vector field $F: \mathbb{R}^{n} \rightarrow \mathbb{R}^{n}$ ) is said to be $\mathbf{r}$-homogeneous of degree $d$ iff for all $\lambda>0$ and for all $x \in \mathbb{R}^{n}$ we have $\lambda^{-d} V\left(\Lambda_{\mathbf{r}} x\right)=V(x)\left(\operatorname{resp} . \lambda^{-d} \Lambda_{\mathbf{r}}^{-1} F\left(\Lambda_{\mathbf{r}} x\right)=F(x)\right)$.

Note that a vector field $F=\left(F_{1}, \ldots, F_{n}\right)$ is $\mathbf{r}$-homogeneous of degree $d$ iff for each $i \in\{1, \ldots, n\}$, the function $F_{i}$ is $\mathbf{r}$-homogeneous of degree $r_{i}+d$. A homogeneous norm is a continuous, positive definite $\mathbf{r}$-homogeneous function of degree 1. An example of $\mathbf{r}$-homogeneous norm is given by

$$
N(x)=\left(\sum_{i=1}^{n}\left|x_{i}\right|^{\rho / r_{i}}\right)^{1 / \rho}, \rho \geq \max _{1 \leq i \leq n} r_{i} .
$$

Being a kind of symmetry, the homogeneity property should be invariant under a change of coordinates. But this is not true for weighted homogeneity. This motivates for a geometric, coordinate-free definition of homogeneity [Khomenuk, 1961, Kawski, 1995].

Definition 3 A vector field $\nu: \mathbb{R}^{n} \rightarrow \mathbb{R}^{n}$ is said to be Euler if $\nu$ is complete, $C^{1}$ and if the origin is Globally Asymptotically Stable (GAS) for $-\nu$. We will always write $\Phi$ the flow of $\nu$.

Definition 4 Let $\nu$ be an Euler vector field. A function $V$ is said to be $\nu$ homogeneous of degree $d$ iff for all $s \in \mathbb{R}$ we have:

$$
e^{-d s} V\left(\Phi^{s}(x)\right)=V(x) .
$$

A vector field $F$ is said to be $\nu$-homogeneous of degree $d$ iff for all $s \in \mathbb{R}$ we have:

$$
e^{-d s}\left(d_{x} \Phi^{s}\right)^{-1} F\left(\Phi^{s}(x)\right)=F(x) .
$$

It is straightforward to check that considering the generalized weight $\mathbf{r}=$ $\left(r_{1}, \ldots, r_{n}\right)$, with $r_{i}>0$ and setting $\nu(x)=r_{1} x_{1} \frac{\partial}{\partial x_{1}}+\ldots+r_{n} x_{n} \frac{\partial}{\partial x_{n}}$ in a fixed basis, a function or a vector field will be $\mathbf{r}$-homogeneous iff it is $\nu$-homogeneous of the same degree.

Proposition $1 A C^{1}$ function $V$ is $\nu$-homogeneous of degree $\mu$ iff $\mathcal{L}_{\nu} V=\mu V$. $A C^{1}$ vector field $F$ is $\nu$-homogeneous of degree $\mu$ iff $[\nu, F]=\mu F$.

The main feature of homogeneous systems (i.e. systems defined by a homogeneous vector field) is related with the following proposition about a kind of symmetry of trajectories, or a commutation property of flows [Kawski, 1995].

Proposition 2 Consider $F \in \operatorname{Lip}\left(\mathbb{R}^{n}\right)$ and denote by $\Psi$ its flow. Then $F$ is $\nu$-homogeneous of degree d iff

$$
\Phi^{s} \circ \Psi^{e^{d s} t}=\Psi^{t} \circ \Phi^{s}, \quad \forall s, t \in \mathbb{R} .
$$


Due to this result, if a homogeneous system is locally asymptotically stable (attractive), then it is GAS.

Example 1 Consider the following scalar system:

$$
\dot{x}=-\frac{a x}{b+|x|},
$$

where $a>0$ and $b>0$ are parameters. Obviously, this system is not homogeneous with respect to Definition 2 . However, it is straightforward to verify using Proposition 1 that it is homogeneous in the sense of Definition 4 with $d=1$ for

$$
\nu(x)=b^{-1} x(b+|x|) .
$$

The origin of this system is locally asymptotically stable (its linearization is stable), and therefore, thanks to homogeneity, the origin is GAS.

\subsection{Scalability and scale invariance}

In this section, $n, m \in \mathbb{N}^{*}$. Consider now a system

$$
\begin{aligned}
& \dot{x}=f(x, u), \\
& y=h(x),
\end{aligned}
$$

where $x \in \mathbb{R}^{n}$ is the state, $u \in \mathbb{U}$ is the input (where $\mathbb{U}$ is the set of admissible inputs, namely a subset of the measurable inputs from $\mathbb{R}$ to $\mathbb{R}^{m}$ ) and $y \in \mathbb{R}$ is the output, $f: \mathbb{R}^{n+m} \rightarrow \mathbb{R}^{n}$ is locally Lipschitz continuous. We assume that $\mathbb{U}$ is invariant with respect to any positive scaling. We also assume that for any $\bar{u} \in \mathbb{R}^{m}$, there exists a unique $\sigma(\bar{u}) \in \mathbb{R}^{n}$ such that $f(\sigma(\bar{u}), \bar{u})=0$. Let us denote by $x\left(t, x_{0}, u\right)$ the current state of the system under input $u$ and with initial condition $x_{0}$. Finally, we denote $y\left(t, x_{0}, u\right)=h\left(x\left(t, x_{0}, u\right)\right)$.

Following [Shoval et al., 2011,Skataric \& Sontag, 2012] let us introduce the main properties under consideration in this note.

Definition 5 The system (2) is said to be positive Scalable (pS) with respect to the inputs from $\mathbb{U}$ if there exists a function $\beta: \mathbb{R}_{+}^{*} \rightarrow \mathbb{R}_{+}^{*}$ such that for any $\bar{u} \in \mathbb{R}^{m}$, any input $u \in \mathbb{U}$ and any $\lambda>0$ :

$$
y(t, \sigma(\lambda \bar{u}), \lambda u)=\beta(\lambda) y(t, \sigma(\bar{u}), u) \quad \forall t \geq 0 .
$$

It is worth to stress that, comparing with [Shoval et al., 2011,Skataric \& Sontag, 2012, Hamadeh et al., 2013], in this work we only consider scalings by a positive $\lambda$, which is the reason why this property is called pS. In [Shoval et al., 2011] a much more general $\mathcal{P}$-invariance property has been considered, where $\mathcal{P}$ is a set of continuous input transformations, while in the present work only multiplication by a constant $\lambda$ is analyzed.

In this definition the constant $\bar{u} \in \mathbb{R}^{m}$ represents the influence of the inputs applied before the instant $t=0$. Hence, the system starts its movement from the equilibrium $\sigma(\bar{u})$ corresponding to that input. The input $u \in \mathbb{U}$ is applied 
for $t \geq 0$ and the scalability property means that if all inputs have been scaled by a $\lambda>0$ (i.e. $\bar{u}$ and $u$ ), then the output of the system is scaled by $\beta(\lambda)$.

If this property is satisfied and the system is excited by two different inputs $u \in \mathbb{U}$ and $u^{\prime} \in \mathbb{U}$, then

$$
\frac{y(t, \sigma(\bar{u}), u)}{y\left(t, \sigma\left(\bar{u}^{\prime}\right), u^{\prime}\right)}=\frac{y(t, \sigma(\lambda \bar{u}), \lambda u)}{y\left(t, \sigma\left(\lambda \bar{u}^{\prime}\right), \lambda u^{\prime}\right)}
$$

for any $\lambda>0$, which means that the scaling factor $\lambda$ is unimportant and only the "ratio" $\frac{u}{u^{\prime}}$ has to be taken into account.

Definition 6 A pS system (2) such that $\beta=1$ is said to admit a positive Fold Change Detection (pFCD) property.

A pFCD system (2) has exactly the same response for an input $u$ applied at the initial condition $\sigma(\bar{u})$ as for an input $\lambda u$ applied at the initial condition $\sigma(\lambda \bar{u})$.

\section{Application of homogeneity}

In the work [Shoval et al., 2011], generic conditions for a dynamical system to have invariant response with respect to symmetries in $\mathcal{P}$ have been proposed (for the case $\beta=1$ ). Homogeneity is a particular kind of symmetry, thus in this section we shall reformulate and partially repeat the results of [Shoval et al., 2011] using homogeneity theory. This development will be used in the next section for investigating the approximate scale invariance property based on the homogeneity framework.

We assume the following homogeneity properties for the system (2).

Assumption 1 There exists an Euler vector field $\nu_{1}: \mathbb{R}^{n} \rightarrow \mathbb{R}^{n}$, whose flow is denoted by $\Phi_{1}$, such that $f\left(\Phi_{1}^{s}(x), e^{s} u\right)=d_{x} \Phi_{1}^{s} f(x, u)$. The function $h$ is $\nu_{1}$-homogeneous of degree $p$.

Remark 1 Note that under these conditions, if for $u=0$ the origin of system (2) is GAS, then (2) is Input-to-State Stable (ISS) [Bernuau et al., 2013a, Bernuau et al., 2013b, Bernuau et al., 2014] (in the case of coordinate-free homogeneity the ISS property depends on characteristics of $\nu$ ).

In this section, we will study the pS property of the system (2) under the class of locally bounded inputs, denoted by $\overline{\mathbb{U}}$, but we will first look at this property for a smaller class of inputs, denoted by $\mathbb{U}$. Let us define this class.

For a given $q \in \mathbb{N}_{+}^{*}$, an admissible $q$-triple is a triple $\left(\phi, g, \xi_{0}\right) \in C^{0}\left(\mathbb{R}^{q}, \mathbb{R}^{m}\right) \times$ $\operatorname{Lip}\left(\mathbb{R}^{q}\right) \times \mathbb{R}^{q}$ such that there exists an Euler vector field $\nu_{2}: \mathbb{R}^{q} \rightarrow \mathbb{R}^{q}$ with flow $\Phi_{2}$ such that $g$ is $\nu_{2}$-homogeneous of degree 0 and $\phi \circ \Phi_{2}^{s}=e^{s} \phi$ for all $s \in \mathbb{R}$ (if $m=1$, then this property means that the function $\phi$ is $\nu_{2}$-homogeneous of degree 1). Remark that no hypothesis is made on $\xi_{0}$. 
We denote $\mathbb{T}_{q}$ the set of admissible $q$-triples. The set of admissible inputs $u$ we consider is given by:

$$
\begin{aligned}
& \mathbb{U}=\left\{u: \mathbb{R} \rightarrow \mathbb{R}^{m}: \exists q>0, \exists\left(\phi, g, \xi_{0}\right) \in \mathbb{T}_{q}:\right. \\
&\left.u(t)=\phi\left(\Xi_{g}^{t}\left(\xi_{0}\right)\right)\right\},
\end{aligned}
$$

where $\Xi_{g}$ is the flow of the system $\dot{\xi}=g(\xi)$. Any triple $\left(\phi, g, \xi_{0}\right) \in \mathbb{T}_{q}$ such that $u(t)=\phi\left(\Xi_{g}^{t}\left(\xi_{0}\right)\right) \in \mathbb{U}$, will be called an associated q-triple for the corresponding $u \in \mathbb{U}$. Therefore, we will consider the class of inputs $\mathbb{U}$ generated by a homogeneous differential equation:

$$
\dot{\xi}(t)=g(\xi(t)), \quad u(t)=\phi(\xi(t))
$$

with the initial condition $\xi_{0} \in \mathbb{R}^{q}$. Thus the scaling property under consideration in this note can be interpreted as the output scalability for a consequent connection of two dynamical systems, when positive rescaling of initial conditions for the first system leads to a corresponding scaling of the output of the second system.

Proposition 3 The set $\mathbb{U}$ is a vector space.

Proof The zero input is clearly an element of $\mathbb{U}$. Consider $u \in \mathbb{U}$ with associated $q$-triple $\left(\phi, g, \xi_{0}\right)$ and $\lambda>0$. There exists $s \in \mathbb{R}$ such that $\lambda=e^{s}$. Therefore $\lambda u(t)=e^{s} \phi\left(\Xi_{g}^{t}\left(\xi_{0}\right)\right)=\phi\left(\Phi_{2}^{s} \circ \Xi_{g}^{t}\left(\xi_{0}\right)\right)=\phi\left(\Xi_{g}^{t} \circ \Phi_{2}^{s}\left(\xi_{0}\right)\right)$. Hence $\lambda u \in \mathbb{U}$ with associated $q$-triple $\left(\phi, g, \Phi_{2}^{s}\left(\xi_{0}\right)\right)$. Moreover $-u \in \mathbb{U}$, with associated $q$-triple $\left(-\phi, g, \xi_{0}\right)$.

If $u_{1} \in \mathbb{U}$ with associated $q_{1}$-triple $\left(\phi_{1}, g_{1}, \theta_{0}\right)$ and homogeneity defined by $\nu_{\theta}$, if $u_{2} \in \mathbb{U}$ with associated $q_{2}$-triple $\left(\phi_{2}, g_{2}, \eta_{0}\right)$ and homogeneity defined by $\nu_{\eta}$, it is straightforward to check that $u_{1}+u_{2} \in \mathbb{U}$ for $q=q_{1}+q_{2}$ and associated $q$-triple $\left(\phi_{1}(\theta)+\phi_{2}(\eta),\left(g_{1}, g_{2}\right),\left(\theta_{0}, \eta_{0}\right)\right)$ and homogeneity defined by $\nu(\theta, \eta)=\left(\nu_{\theta}(\theta), \nu_{\eta}(\eta)\right)$.

\subsection{Homogeneity conditions for pS property}

For an initial condition $x_{0} \in \mathbb{R}^{n}$ and an input $u \in \mathbb{U}$, consider an associated $q$-triple $\left(\phi, g, \xi_{0}\right)$ and define:

$$
F\left(\begin{array}{l}
x \\
\xi
\end{array}\right)=\left(\begin{array}{c}
f(x, \phi(\xi)) \\
g(\xi)
\end{array}\right) .
$$

We denote $\Psi$ the flow of $F$. Denote $p_{1}: \mathbb{R}^{n} \times \mathbb{R}^{q} \rightarrow \mathbb{R}^{n}$ and $p_{2}: \mathbb{R}^{n} \times$ $\mathbb{R}^{q} \rightarrow \mathbb{R}^{q}$ the first and the second canonical projections respectively. We have $y\left(t, x_{0}, u\right)=h\left(p_{1} \circ \Psi^{t}\left(x_{0}, \xi_{0}\right)\right)$.

Let us denote $\nu\left(\begin{array}{l}x \\ \xi\end{array}\right)=\left(\begin{array}{l}\nu_{1}(x) \\ \nu_{2}(\xi)\end{array}\right)$ and $\Phi$ the flow of $\nu$. 
Lemma 1 If Assumption 1 is verified, the following properties hold:

1. The vector field $F$ is $\nu$-homogeneous of degree 0 .

2. Denote $\Phi_{1}^{s}=p_{1} \circ \Phi^{s}$, then $\Phi_{1}^{s}(\sigma(\bar{u}))=\sigma\left(e^{s} \bar{u}\right)$.

3. Denote $\Psi_{2}^{t}=p_{2} \circ \Psi^{t}$, then the mapping $\Psi_{2}$ is the flow of $g$ and $\Psi_{2}^{t} \circ \Phi_{2}^{s}=$ $\Phi_{2}^{s} \circ \Psi_{2}^{t}$.

Proof The vector field $F$ is $\nu$-homogeneous of degree 0 iff $F \circ \Phi^{s}=d \Phi^{s} F$ :

$$
\left(\begin{array}{c}
f\left(\Phi_{1}^{s}(x), \phi\left(\Phi_{2}^{s}(\xi)\right)\right) \\
g\left(\Phi_{2}^{s}(\xi)\right)
\end{array}\right)=\left(\begin{array}{c}
d_{x} \Phi_{1}^{s} f(x, \phi(\xi)) \\
d_{\xi} \Phi_{2}^{s} g(\xi)
\end{array}\right)
$$

which is given by Assumption 1 and the definition of an associate triple for $\mathbb{U}$.

We have $\frac{d}{d s}\left[p_{1} \circ \Phi^{s}\right]=p_{1} \circ \nu\left(\Phi^{s}\right)=\nu_{1}\left(p_{1} \circ \Phi^{s}\right)$. Similarly, $\frac{d}{d t} p_{2} \circ \Psi^{t}=$ $p_{2} F\left(\Psi^{t}\right)=g\left(p_{2} \circ \Psi^{t}\right)$. The vector field $g$ is $\nu_{2}$-homogeneous, then the identity $\Psi_{2}^{t} \circ \Phi_{2}^{s}=\Phi_{2}^{s} \circ \Psi_{2}^{t}$ follows.

Let $\bar{u}=\phi(\bar{\xi})$ for some $\bar{u} \in \mathbb{R}^{m}$ and $\bar{\xi} \in \mathbb{R}^{q}$, then we have $0=f\left(\sigma\left(e^{s} \bar{u}\right), e^{s} \bar{u}\right)=$ $f\left(\Phi_{1}^{s} \circ \Phi_{1}^{-s} \circ \sigma\left(e^{s} \bar{u}\right), \phi\left(\Phi_{2}^{s}(\bar{\xi})\right)=d_{x} \Phi_{1}^{s} f\left(\Phi_{1}^{-s} \circ \sigma\left(e^{s} \bar{u}\right), \phi(\bar{\xi})\right)\right.$. Since $d_{x} \Phi_{1}^{s}$ is a nonsingular matrix, then $f\left(\Phi_{1}^{-s} \circ \sigma\left(e^{s} \bar{u}\right), \bar{u}\right)=0$ and $\sigma(\bar{u})=\Phi_{1}^{-s} \circ \sigma\left(e^{s} \bar{u}\right)$ provided that $\sigma(\bar{u})$ is the unique equilibrium of the system (2) for the constant input $\bar{u}$.

Theorem 1 Under Assumption 1, the system (2) is $p S$ with respect to inputs from $\mathbb{U}$ and $\beta(\lambda)=\lambda^{p}$. Moreover, if $p=0$, then the system admits $p F C D$ property.

Proof Set $\bar{u} \in \mathbb{R}^{m}$, an input $u$ and $\lambda>0$. There exists an unique $s \in \mathbb{R}$ such that $\lambda=e^{s}$, and a $\xi_{0}$ which determines $u$. We have $u(t)=\phi\left(\Psi_{2}^{t}\left(\xi_{0}\right)\right)$ and therefore $\lambda u(t)=e^{s} \phi\left(\Psi_{2}^{t}\left(\xi_{0}\right)\right)=\phi\left(\Phi_{2}^{s} \circ \Psi_{2}^{t}\left(\xi_{0}\right)\right)=\phi\left(\Psi_{2}^{t} \circ \Phi_{2}^{s}\left(\xi_{0}\right)\right)$. This leads to:

$$
\begin{aligned}
y(t, \sigma(\lambda \bar{u}), \lambda u) & =h\left(p_{1} \circ \Psi^{t}\left(\sigma(\lambda \bar{u}), \Phi_{2}^{s}\left(\xi_{0}\right)\right)\right) \\
& =h\left(p_{1} \circ \Psi^{t}\left(\Phi_{1}^{s}(\sigma(\bar{u})), \Phi_{2}^{s}\left(\xi_{0}\right)\right)\right) \\
& =h\left(p_{1} \circ \Psi^{t} \circ \Phi^{s}\left(\sigma(\bar{u}), \xi_{0}\right)\right) \\
& =h\left(p_{1} \circ \Phi^{s} \circ \Psi^{t}\left(\sigma(\bar{u}), \xi_{0}\right)\right) \\
& =h\left(\Phi_{1}^{s} \circ \Psi^{t}\left(\sigma(\bar{u}), \xi_{0}\right)\right) \\
& =e^{p s} h\left(\Psi^{t}\left(\sigma(\bar{u}), \xi_{0}\right)\right) \\
& =\lambda^{p} y(t, \sigma(\bar{u}), u) .
\end{aligned}
$$

Corollary 1 Under Assumption 1, the system (2) is $p S$ with respect to polynomials inputs.

Proof Let $u(t)=\sum_{i=0}^{q-1} \frac{a_{i}}{i !} t^{i}$ be a polynomial input of degree $q-1$. The $q-$ triple $\left(\phi, g, \xi_{0}\right)$ defined by $\phi(\xi)=\sum_{i=0}^{q-1} a_{q-1-i} \xi^{i}\left(\right.$ where $\left.\xi=\left(\xi^{0}, \ldots, \xi^{q-1}\right)\right)$, $g(\xi)=\left(\xi^{1}, \ldots, \xi^{q-1}, 0\right)$ and $\xi_{0}=(0,0, \ldots, 0,1)$ is admissible. Indeed, it is straightforward to check that $u(t)=\phi\left(\Xi_{g}^{t}\left(\xi_{0}\right)\right)$ and $\nu_{2}(\xi)=\xi$.

Theorem 2 Under Assumption 1, the system (2) is $p S$ with respect to locally bounded inputs. 
To prove this theorem, we will first need the following lemma.

Lemma 2 Let $u$ be a bounded input defined on $[0, T]$. Suppose that there exists a sequence $\left(u_{n}\right)_{n \geq 1}$ of bounded inputs converging to $u$ in $\mathcal{L}^{1}[0, T]$ (i.e. $\int_{0}^{T}\left|u_{n}(t)-u(t)\right| d t=\left\|u_{n}(t)-u(t)\right\|_{\mathcal{L}^{1}[0, T]} \rightarrow 0$ when $\left.n \rightarrow+\infty\right)$ such that $\left\|u_{n}\right\|_{\mathcal{L}^{\infty}[0, T]} \leq 1+\|u\|_{\mathcal{L}^{\infty}[0, T]}$. Let $x$ be the trajectory of (2) with input $u$ and initial condition $x(0)=x_{0}$ and $x_{n}$ be the trajectory of (2) with input $u_{n}$ and initial condition $x_{n}(0)=x_{0}$. Then $x_{n}$ converges to $x$ uniformly on $[0, T]$.

Proof Since $x$ is continuous, then the set $\{x(t): t \in[0, T]\}$ is compact and thus the set $X=\{x(t): t \in[0, T]\}+B$ is also compact, where $B$ is the closed unit ball in $\mathbb{R}^{n}$. Similarly, the set $U=\left(1+\|u\|_{\mathcal{L}^{\infty}[0, T]}\right) B^{\prime}$ is compact, where $B^{\prime}$ is the closed unit ball in $\mathbb{R}^{m}$. Denoting $K=X \times U$, the set $K$ is compact. The vector field $f$ is locally Lipschitz continuous, then we can choose a uniform Lipschitz constant $C$ on $K$. Hence:

$$
\begin{gathered}
\forall\left(\bar{x}_{1}, \bar{u}_{1}\right) \in K, \forall\left(\bar{x}_{2}, \bar{u}_{2}\right) \in K: \\
\left|f\left(\bar{x}_{1}, \bar{u}_{1}\right)-f\left(\bar{x}_{2}, \bar{u}_{2}\right)\right| \leq C\left(\left|\bar{x}_{1}-\bar{x}_{2}\right|+\left|\bar{u}_{1}-\bar{u}_{2}\right|\right) .
\end{gathered}
$$

We have $x_{n}(0) \in X$, thus by continuity there exists $T_{n}>0$ such that $x_{n}(t) \in$ $X$ for all $t \in\left[0, T_{n}\right]$. Without loss of generality, we assume $T_{n} \leq T$ and $T_{n}=\inf \left\{t>0:\left|x(t)-x_{n}(t)\right|>1\right\}$. By continuity, if $T_{n}<T$, we have $\left|x\left(T_{n}\right)-x_{n}\left(T_{n}\right)\right|=1$. Moreover, the hypothesis on $u_{n}$ implies that $u_{n}(t) \in U$ for all $t \in[0, T]$. Hence for all $t \in\left[0, T_{n}\right],\left(x_{n}(t), u_{n}(t)\right) \in K$. Denoting $\Delta(t)=$ $\left|x(t)-x_{n}(t)\right|^{2}$, we have for all $t \in\left[0, T_{n}\right]$ :

$$
\begin{aligned}
\frac{d \Delta}{d t} & =2\left\langle x-x_{n}, f(x, u)-f\left(x_{n}, u_{n}\right)\right\rangle \\
& \leq 2\left|x-x_{n}\right|\left|f(x, u)-f\left(x_{n}, u_{n}\right)\right| \\
& \leq 2 C\left|x-x_{n}\right|\left(\left|u-u_{n}\right|+\left|x-x_{n}\right|\right) \\
& \leq 2 C \Delta+2 C \sqrt{\Delta}\left|u-u_{n}\right| .
\end{aligned}
$$

Setting $z(t)=\Delta e^{-2 C t}$, we get $\dot{z} \leq 2 C \sqrt{z} e^{-C t}\left|u-u_{n}\right|$. Integrating this differential inequality, we get:

$$
\sqrt{z(t)} \leq C \int_{0}^{t} e^{-C \tau}\left|u(\tau)-u_{n}(\tau)\right| d \tau \leq C\left\|u-u_{n}\right\|_{\mathcal{L}^{1}[0, T]},
$$

since $z(0)=\Delta(0)=0$. We finally find that $\Delta(t) \leq C^{2} e^{2 C T}\left\|u-u_{n}\right\|_{\mathcal{L}^{1}[0, T]}^{2}$. Thus for all $\epsilon>0$ and $n$ large enough, $\sup _{t \in\left[0, T_{n}\right]} \Delta(t)<\epsilon$, which proves that $T_{n}=T$ and that $x_{n}$ converges to $x$ uniformly on $[0, T]$.

Proof of Theorem 2 Fix $T>0$. For an input $u$ and an initial condition $x_{0}$, we denote by $y\left(t, x_{0}, u\right)$ the output of the system (2) at time $t \in[0, T]$. Fix $\lambda>0$, $\bar{u} \in \mathbb{R}^{m}$ and consider for $t \geq 0$ the function $I$ defined by:

$$
I(t, u)=y(t, \sigma(\lambda \bar{u}), \lambda u)-\lambda^{p} y(t, \sigma(\bar{u}), u) .
$$


We shall first prove that the pS property holds for continuous inputs, that is, for any given continuous input $u$ and any time $t \geq 0$, we have $I(t, u)=0$. Fix $T>0$. By the theorem of Weierstrass, any continuous function defined on a segment $[0, T]$ can be uniformly approximated by polynomials. Therefore, there exists a sequence $\left(u_{n}\right)$ of polynomial inputs converging to $u$ uniformly on $[0, T]$. Thus there exists an index $N>0$ such that for all $n \geq N\left\|u_{n}\right\|_{\mathcal{L}^{\infty}[0, T]} \leq$ $1+\|u\|_{\mathcal{L}^{\infty}[0, T]}$, and $\left\|u-u_{n}\right\|_{\mathcal{L}^{1}[0, T]} \leq T\left\|u-u_{n}\right\|_{\mathcal{L}^{\infty}[0, T]} \rightarrow 0$. With the notation of Lemma 2 , we find that $x_{n}$ converges to $x$ uniformly on $[0, T]$. We claim that $y\left(t, x_{0}, u_{n}\right)$ converges to $y\left(t, x_{0}, u\right)$ uniformly on $[0, T]$. Indeed, $h$ is continuous and therefore uniformly continuous on the compact set $X$. Together with the uniform convergence of $x_{n}$, this proves the claim.

By Theorem 1 we have $I(t, v)=0$ for all $v \in \mathbb{U}$ and then $I\left(t, u_{n}\right)=0$. We have:

$$
\begin{aligned}
|I(t, u)|= & \left|I(t, u)-I\left(t, u_{n}\right)\right| \\
\leq & \left|y(t, \sigma(\lambda \bar{u}), \lambda u)-y\left(t, \sigma(\lambda \bar{u}), \lambda u_{n}\right)\right| \\
& +\lambda^{p}\left|y(t, \sigma(\bar{u}), u)-y\left(t, \sigma(\bar{u}), u_{n}\right)\right| .
\end{aligned}
$$

By the claim, we conclude that for all $\epsilon>0,|I(t, u)|<\epsilon$, and hence $I(t, u)=0$ for all $t \in[0, T]$.

To prove it for locally bounded inputs, we will apply the same method. Fix a locally bounded input $u \neq 0$ and $T>0$. There exists a sequence of continuous inputs $\left(u_{n}\right)$ such that $\left\|u-u_{n}\right\|_{\mathcal{L}^{1}[0, T]} \rightarrow 0$. Let us denote, for $\bar{u} \in \mathbb{R}^{m}$, sat $(\bar{u})=\bar{u}$ if $|\bar{u}|<1$ and $\operatorname{sat}(\bar{u})=\frac{\bar{u}}{|\bar{u}|}$ otherwise. Consider $\bar{u}_{n}(t)=(1+$ $\left.\|u\|_{\mathcal{L}^{\infty}[0, T]}\right) \operatorname{sat}\left(\frac{u_{n}(t)}{1+\|u\|_{\mathcal{L}^{\infty}[0, T]}}\right)$. The sequence $\left(\bar{u}_{n}\right)$ is bounded by $1+\|U\|_{\mathcal{L}^{\infty}[0, T]}$ and converges to $u$ in $\mathcal{L}^{1}[0, T]$. Therefore, with the notations of the Lemma 2 , we find that $x_{n}$ converges to $x$ uniformly on $[0, T]$, and we conclude that $I(t, u)=0$ similarly.

We proved that $I(t, u)=0$ for any $t \in[0, T]$. But the value $T$ being chosen arbitrarily, we finally find that for any $t \geq 0$ we have $I(t, u)=0$ which proves the theorem.

\subsection{The case of constant inputs}

Let $\mathbb{U}$ be the class of constant inputs, in this case

$$
g(\xi)=0, \quad \phi(\xi)=\xi
$$

then $\nu_{2}(\xi)=\xi$. Following the result of Lemma 1, to verify Assumption 1 it is equivalent to check that the vector field $F(x, u)=\left[f(x, u)^{T} 0\right]^{T}$ is homogeneous for $\nu(x, u)=\left[\nu_{1}(x)^{T} u\right]^{T}$ of degree $d=0$.

Consider examples of such a case study.

Example 2 Consider a linear system with

$$
f(x, u)=A x+b u, y=c^{T} x,
$$


where $A$ is an invertible matrix of dimension $n \times n, b$ and $c$ are some vectors in $\mathbb{R}^{n}$. This system is (weighted) homogeneous with $d=0$ and $p=1$ and, hence, it admits the pS property with respect to constant inputs. For $y=\operatorname{sign}\left(c^{T} x\right)$ the system has pFCD property.

Example 3 Take

$$
f(x, u)=-\frac{a x}{1+|x|}+\frac{e^{-|x|}}{1+|x|} u, h(x)=e^{p|x|} x^{p},
$$

where $a>0, p>0$, then $F$ and $h$ are homogeneous for $\nu_{1}(x)=\frac{x}{1+|x|}$ of degrees $d=0$ and $p$ respectively. Thus, this nonlinear system is $\mathrm{pS}$ with respect to constant inputs.

\subsection{The case of different rates of convergence}

Let us discuss the case of nonzero degree $d$ of $F$. In this case, the image of the trajectories in the state space stay the same, but the convergence time scale is modified. In other words, in this case there exist functions $\beta: \mathbb{R}_{>0} \rightarrow \mathbb{R}_{>0}$ and $\gamma: \mathbb{R}_{>0} \rightarrow \mathbb{R}_{>0}$ such that for any $\bar{u} \in \mathbb{R}^{m}$, any input $u \in \mathbb{U}$ and any $\lambda>0$ :

$$
y(t, \sigma(\lambda \bar{u}), \lambda u)=\beta(\lambda) y(\gamma(\lambda) t, \sigma(\bar{u}), u) \quad \forall t \geq 0 .
$$

Let us evaluate the rate of convergence. Consider a homogeneous norm $N$, that is a continuous positive definite $\nu$-homogeneous function of degree 1 . Such a function always exists (it is a direct corollary of [Bacciotti \& Rosier, 2005][Theorem 5.13 ] applied to $-\nu$, which is $\nu$-homogeneous of degree 0 ). Pick an $\epsilon>0$, then we have:

$$
N\left(\Psi^{t}\left(0, e^{s} u_{0}\right)\right) \leq \epsilon \Leftrightarrow N\left(\Psi^{e^{d s}} t\left(0, u_{0}\right)\right) \leq e^{-s} \epsilon .
$$

Therefore, denoting $T_{\epsilon}(x, u)=\inf \left\{t \geq 0 \forall \tau \geq t: N\left(\Psi^{\tau}(x, u)\right) \leq \epsilon\right\}$, we have $T_{\epsilon}\left(0, e^{s} u_{0}\right)=T_{e^{-s} \epsilon}\left(0, u_{0}\right)$, and the rate of time response of the system is related to the input scaling $e^{s}$.

\section{Local homogeneity}

Previously we assumed that the vector field $F$ was homogeneous, which is a rather restrictive hypothesis. This assumption may be relaxed using local homogeneity [Zubov, 1958, Andrieu et al., 2008, Efimov \& Perruquetti, 2010].

Definition 7 Let $F_{0}$ be a vector field such that

$$
e^{-d s}\left(d_{x} \Phi^{s}\right)^{-1} F\left(\Phi^{s}(x)\right)
$$

converges to $F_{0}(x)$ uniformly on compact sets as $s \rightarrow-\infty(s \rightarrow+\infty)$. Then $F_{0}$ is called the local $\nu$-homogeneous approximation of degree $d$ at 0 (at $\left.+\infty\right)$ of $F$. 
The local $\nu$-homogeneous approximation $F_{0}$ of degree $d$ is always homogeneous of degree $d$. Only one degree can give us an interesting approximation. An easy verification shows that, if for a given $d, F_{0}$ is non-vanishing, then all others approximations are either vanishing or not defined. When such a non-vanishing $\nu$-homogeneous approximation $F_{0}$ exists, we call it the $\nu$-homogenization of $F$. It is possible to show that if $F_{0}$ is the local $\nu$-homogeneous approximation of degree $d$ at $0($ at $+\infty)$ of $F$, then for any $\epsilon>0$ there exists $s_{\epsilon}<0\left(s_{\epsilon}>0\right)$ such that

$$
\begin{gathered}
\sup _{y \in \mathbb{S}_{N}, \operatorname{sign}\left(s_{\epsilon}\right) s \geq\left|s_{\epsilon}\right|}|w(s, y)| \leq \epsilon, \\
w(s, y)=e^{-d s}\left(d_{y} \Phi^{s}\right)^{-1} F\left(\Phi^{s}(y)\right)-F_{0}(y),
\end{gathered}
$$

where $\mathbb{S}_{N}=\left\{x \in \mathbb{R}^{n}: N(x)=1\right\}$, with $N$ a homogeneous norm. Further in this section the case of weighted homogeneity with $\nu(x)=\operatorname{diag}\left(r_{1} x_{1}, \ldots, r_{n} x_{n}\right)$ for some $r_{i} \in \mathbb{R}_{+}^{*}, 1 \leq i \leq n$ is considered (it is the most useful case for a local approximation calculation). In this case for any $x \in \mathbb{R}^{n} \backslash\{0\}$ there exist $s \in \mathbb{R}$ and $y \in \mathbb{S}_{\nu}$ such that $x=\Phi^{s}(y)$ and

$$
\begin{aligned}
\left|F(x)-F_{0}(x)\right| & =\left|e^{d s} d_{y} \Phi^{s} w(s, y)\right| \\
& =\left|e^{d s} \operatorname{diag}\left(e^{r_{1} s}, \ldots, e^{r_{n} s}\right) w(s, y)\right| \\
& \leq \gamma(s)|w(s, y)|, \\
\gamma(s) & = \begin{cases}e^{\left(r_{\min }+d\right) s} & s \leq 0, \\
e^{\left(r_{\max }+d\right) s} & s>0,\end{cases}
\end{aligned}
$$

where $r_{\min }=\min _{1 \leq i \leq n} r_{i}$ and $r_{\max }=\max _{1 \leq i \leq n} r_{i}$. The following stability properties are satisfied [Bacciotti \& Rosier, 2005,Zubov, 1958, Andrieu et al., 2008]:

Theorem 3 Let $F$ be a continuous vector field and assume that there exists a non-zero vector field $F_{0}$ being its local approximation of degree $d$ at 0 (at $+\infty)$. If the origin is $G A S$ for $F_{0}$, then it is locally asymptotically stable for $F$ (and then $F$ has bounded trajectories).

The local approximation provides local information on the behavior of the system and can be defined for any finite value $s_{0} \in(-\infty,+\infty)$ [Efimov \& Perruquetti, 2010]. It can also be used to investigate the scale invariance property, although only locally. In the paper [Skataric \& Sontag, 2012], it has been observed that the class of systems for which the scale invariance property exactly holds is very limited, and the notion of approximate scale invariance has been introduced. We will use the following definitions of that notion here.

Definition 8 For an $\varepsilon \in \mathbb{R}_{+}$, the system (2) is said to be $\varepsilon$-positive Approximately Scalable $(\varepsilon-\mathrm{pAS})$ with respect to the inputs from $\mathbb{U}$ if there exists a function $\beta: \mathbb{R}_{+}^{*} \rightarrow \mathbb{R}_{+}^{*}$ such that for any $\bar{u} \in \mathbb{R}^{m}$, any input $u \in \mathbb{U}$ and any $\lambda>0$ :

$$
\sup _{t \geq 0}|y(t, \sigma(\lambda \bar{u}), \lambda u)-\beta(\lambda) y(t, \sigma(\bar{u}), u)| \leq \varepsilon .
$$


Definition 9 The system (2) is said to be logarithmic $\varepsilon$-pAS with respect to the inputs from $\mathbb{U}$ if for any $\bar{u} \in \mathbb{R}^{m}$, any input $u \in \mathbb{U}$ and any $\lambda>0$ there exists $\beta: \mathbb{R}_{+}^{*} \rightarrow \mathbb{R}_{+}^{*}$ such that

$$
\sup _{t \geq 0}\left|\frac{y(t, \sigma(\lambda \bar{u}), \lambda u)}{\beta(\lambda) y(t, \sigma(\bar{u}), u)}-1\right| \leq \varepsilon .
$$

These properties can also be defined for $\bar{u} \in \overline{\mathcal{U}} \subset \mathbb{R}^{m}, u \in \mathcal{U} \subset \mathbb{U}$ and $\lambda \in \Lambda \subset \mathbb{R}_{+}^{*}$.

The difference between $\varepsilon$-pAS and logarithmic $\varepsilon$-pAS relies in the fact that the former one is better suited for the analysis of approximate scalability at the origin (for small values of $|y|$ ), while the latter is better adapted for the scalability investigations for big values of $y$.

The approximate scale invariance property has been analyzed in [Skataric \& Sontag, 2012] applying time-separation and the linearization techniques. In this work we will use the local homogeneity framework for the same purpose. An advantage of this method is that it provides a way of approximating the system at infinity and not only at the origin as with the linearization.

4.1 Relation between trajectories of $F$ and $F_{0}$

First, let us establish the conditions under which the trajectories of a locally Lipschitz system

$$
\dot{x}=F(x),
$$

and its $\nu$-approximation

$$
\dot{x}=F_{0}(x),
$$

starting from the same initial conditions, stay close. More precisely, denote $\Psi$ and $\Psi_{0}$ the flows of $F$ and $F_{0}$ respectively and select an $\varepsilon>0$; we are looking for conditions under which $\left|\Psi^{t}(x)-\Psi_{0}^{t}(x)\right| \leq \varepsilon$ for a given $x \in \mathbb{R}^{n}$ and for some time interval $t \in[0, T]$. Define $T_{x}^{r}=\left\{t \geq 0: \Psi^{t}(x) \in \mathcal{N}_{r}\right\}$, where $\mathcal{N}_{r}=\left\{x \in \mathbb{R}^{n}: \operatorname{sign}(r) N(x) \geq \operatorname{sign}(r) e^{r}\right\}$ (let us recall that $N$ is a $\nu$ homogeneous norm, that is a continuous and positive definite $\nu$-homogeneous function of degree 1). Note that the set $T_{x}^{r}$ can be empty if $\Psi^{t}(x) \notin \mathcal{N}_{r}$ for all $t \geq 0$.

Lemma 3 Let $\varepsilon>0$ be given and $F_{0}$ be the local $\nu$-homogeneous approximation of degree d at $0(a t+\infty)$ of $F$. Let $F_{0}$ be a $C^{1}$ vector field and there exist matrices $A_{k} \in \mathbb{R}^{n \times n}, 1 \leq k \leq K$ for some $K \in \mathbb{N}^{*}$ such that for all $x \in \mathbb{R}^{n}$

$$
\begin{gathered}
\operatorname{Jac} F_{0}(x) \in C o\left(A_{1}, \ldots, A_{K}\right), \\
A_{k}^{T} P+P A_{k} \leq-Q \quad \forall 1 \leq k \leq K
\end{gathered}
$$


for some matrices $P=P^{T}>0, Q=Q^{T}>0$. Then for all $x \in \mathcal{N}_{s_{\varepsilon / \rho}}$,

$$
\begin{gathered}
\left|\Psi^{t}(x)-\Psi_{0}^{t}(x)\right| \leq \varepsilon \kappa(x), \\
\kappa(x)=\max \left\{\xi \circ N(x)^{d+r_{\min }}, \xi \circ N(x)^{d+r_{\max }}\right\}
\end{gathered}
$$

while $t \in T_{x}^{s_{\varepsilon / \rho}}$, where $\rho=2 \sqrt{\frac{\lambda_{\max }^{3}(P)}{\lambda_{\min }^{2}(Q) \lambda_{\min }(P)}}$ and $\xi$ is a function from $\mathcal{K}_{\infty}$.

The condition that the Jacobian matrix of $F_{0}$ belongs to a convex hull of matrices (6) is widely used in the theory of convergent systems [Pavlov et al., 2004] (together with the stability condition for all matrices in the hull) or for the incremental stability analysis [Ingalls \& Sontag, 2002].

Proof Since $F_{0}$ is homogeneous, the fulfillment of the condition (6) implies that all trajectories are converging [Pavlov et al., 2004,Ingalls \& Sontag, 2002], then the origin is GAS for (5) [Bacciotti \& Rosier, 2005]. By Theorem 3, in this case $F$ has also some stability properties being close to the origin or at infinity. In addition, for an $x \in \mathcal{N}_{s_{\epsilon}}$, if $\epsilon$ has been selected sufficiently small, then $\Psi^{t}(x)$ is bounded for all $t \geq 0$, i.e. there is a function $\xi$ from class $\mathcal{K}_{\infty}$ such that $N \circ \Psi^{t}(x) \leq \xi \circ N(x)$ for all $t \geq 0$.

Consider the discrepancy $e(t, x)=\Psi^{t}(x)-\Psi_{0}^{t}(x)$ of trajectories of the systems (4) and (5), then

$$
\begin{aligned}
& \dot{e}(t, x)=F_{0}\left(\Psi^{t}(x)\right)-F_{0}\left(\Psi_{0}^{t}(x)\right)+\delta(t, x), \\
& \delta(t, x)=F\left(\Psi^{t}(x)\right)-F_{0}\left(\Psi^{t}(x)\right) .
\end{aligned}
$$

Since $F_{0}$ is the local $\nu$-homogeneous approximation of $F$, then for any $\epsilon>0$ there is $s_{\epsilon} \in \mathbb{R}$ such that $|\delta(t, x)| \leq \epsilon \gamma \circ \ln \circ \xi \circ N(x)$ for all $t \in T_{x}^{s_{\epsilon}}$ (i.e. $t \geq 0$ such that $\left.\Psi^{t}(x) \in \mathcal{N}_{s_{\epsilon}}\right)$. Take a candidate of Lyapunov function $V(e)=e^{T} P e$, then

$$
\begin{aligned}
\dot{V}= & 2 e^{T} P\left[F_{0}\left(\Psi^{t}(x)\right)-F_{0}\left(\Psi_{0}^{t}(x)\right)\right]+ \\
& +2 e^{T} P \delta(t, x) .
\end{aligned}
$$

Define $Z(\lambda)=e^{T} P F_{0}\left(\Psi_{0}^{t}(x)+\lambda e\right)$, then $e^{T} P\left[F_{0}\left(\Psi^{t}(x)\right)-F_{0}\left(\Psi_{0}^{t}(x)\right)\right]=Z(1)-$ $Z(0)$ and

$$
\dot{V}=2[Z(1)-Z(0)]+2 e^{T} P \delta(t, x) .
$$

By the Mean Value Theorem $Z(1)-Z(0)=\frac{d Z(\tilde{\lambda})}{d \tilde{\lambda}}$ for some $\tilde{\lambda} \in[0,1]$, then $\frac{d Z(\tilde{\lambda})}{d \tilde{\lambda}}=e^{T} P A(t, x) e$ for $A(t, x)=\left.\operatorname{Jac} F_{0}(\xi)\right|_{\xi=\Psi_{0}^{t}(x)+\tilde{\lambda} e(t, x)}$ and

$$
\dot{V}=e^{T}\left[A^{T}(t, x) P+P A(t, x)\right] e+2 e^{T} P \delta(t, x) .
$$


Note that $A(t, x) \in C o\left(A_{1}, \ldots, A_{K}\right)$, thus $A(t, x)=\sum_{k=1}^{K} \alpha_{k}(t, x) A_{k}, \sum_{k=1}^{K} \alpha_{k}(t, x)=$ 1 and

$$
\begin{aligned}
\dot{V}= & e^{T}\left[\sum_{k=1}^{K} \alpha_{k}(t, x) A_{k}^{T} P+P \sum_{k=1}^{K} \alpha_{k}(t, x) A_{k}\right] e \\
& +2 e^{T} P \delta(t, x) \\
= & e^{T} \sum_{k=1}^{K} \alpha_{k}(t, x)\left[A_{k}^{T} P+P A_{k}\right] e \\
& +2 e^{T} P \delta(t, x) \\
\leq & -e^{T} Q e+2 e^{T} P \delta(t, x) \\
\leq & -0.5 e^{T} Q e+2 \delta(t, x)^{T} P Q^{-1} P \delta(t, x) .
\end{aligned}
$$

Finally we obtain for all $t \geq 0$

$$
\begin{aligned}
|e(t, x)| & \leq e^{-0.25 \frac{\lambda_{\min }(Q)}{\lambda_{\max }(P)} t} \sqrt{\frac{\lambda_{\max }(P)}{\lambda_{\min }(P)}}|e(0, x)| \\
& +2 \sqrt{\frac{\lambda_{\max }^{3}(P)}{\lambda_{\min }^{2}(Q) \lambda_{\min }(P)}} \sup _{0 \leq \tau \leq t}|\delta(\tau, x)| .
\end{aligned}
$$

Since $e(0, x)=0$, then $|e(t, x)| \leq \rho \sup _{0 \leq \tau \leq t}|\delta(\tau, x)| \leq \varepsilon \kappa(x)$ as long as $t \in$ $T_{x}^{s_{\varepsilon / \rho}}$, i.e. $\Psi^{t}(x) \in \mathcal{N}_{s_{\varepsilon / \rho}}$.

If $F_{0}$ is the local $\nu$-homogeneous approximation at 0 of $F$, then it is possible to select $\varepsilon>0$ sufficiently small in such a way that $\xi \circ N(x) \leq 1$, then $\left|\Psi^{t}(x)-\Psi_{0}^{t}(x)\right| \leq \varepsilon$ while trajectories stay in $\mathcal{N}_{s_{\varepsilon / \rho}}$. For the approximation at $+\infty$ the situation is more complicated.

\subsection{Application of local homogeneity for pAS analysis}

The main idea of this section is to combine the results of Theorem 1 and Lemma 3.

In this subsection consider the system in (2) for $h(x)=x_{i}$ with some index $1 \leq i \leq n$ (i.e. $p=1$ ) and an input $u \in \mathbb{U}$, which has an associated triple $\left(\phi, g, \xi_{0}\right)$.

Corollary 2 For the closed loop system (4) with $F$ defined in (3), let $F_{0}=$ $\left[f_{0}^{T} g^{T}\right]^{T}$ be $C^{1}$ local $\nu$-homogeneous approximation of degree 0 at $0($ at $+\infty)$ of $F$ for $\nu=\left(\nu_{1}^{T} \nu_{2}^{T}\right)^{T}$ for some Euler vector field $\nu_{1}$ (the vector field $g$ is $\nu_{2}$-homogeneous). Let there exist matrices $A_{k} \in \mathbb{R}^{n \times n}, 1 \leq k \leq K$ for some $K \in \mathbb{N}^{*}$ such that for all $x \in \mathbb{R}^{n}$ and $u \in \mathbb{U}$

$$
\begin{aligned}
& \operatorname{Jac} f_{0}(x, u(t)) \in C o\left(A_{1}, \ldots, A_{K}\right), \\
& A_{k}^{T} P+P A_{k} \leq-Q \quad \forall 1 \leq k \leq K
\end{aligned}
$$


for some matrices $P=P^{T}>0, Q=Q^{T}>0$. Then for all $x \in \mathcal{N}_{s_{\varepsilon / \rho}}$, where $\rho$ is defined as in Lemma 3,

$$
\begin{gathered}
\left|\Psi^{t}\left(x, \xi_{0}\right)-\Psi_{0}^{t}\left(x, \xi_{0}\right)\right| \leq \varepsilon \kappa\left(x, \xi_{0}\right), \\
\kappa\left(x, \xi_{0}\right)=\max \left\{\xi \circ N\left(x, \xi_{0}\right)^{r_{\min }}, \xi \circ N\left(x, \xi_{0}\right)^{r_{\max }}\right\}
\end{gathered}
$$

while $t \in T_{x}^{s_{\varepsilon / \rho}}$.

Therefore the system (2) has $2 \varepsilon \kappa\left(x, \xi_{0}\right)$-pAS property with $\beta(\lambda)=\lambda$ if approximation at the origin is considered or the logarithmic $2 \varepsilon \kappa\left(x, \xi_{0}\right)$-pAS property for the case of $\nu$-homogeneous approximation at $+\infty$ if $\lambda y(t, \sigma(\bar{u}), u) \geq \eta>1$ in $\mathcal{N}_{s_{\varepsilon / \rho}}$ and $T_{\sigma(\bar{u})}^{s_{\varepsilon / \rho}}=\mathbb{R}_{+}$. Indeed, without losing generality assume that $\lambda>1$ then:

$$
\begin{aligned}
& \sup _{t \geq 0}\left|\frac{y(t, \sigma(\lambda \bar{u}), \lambda u)}{\lambda y(t, \sigma(\bar{u}), u)}-1\right| \\
&=\sup _{t \geq 0}\left|\frac{\Psi_{i}^{t}\left(\lambda x, \lambda \xi_{0}\right)}{\lambda \Psi_{i}^{t}\left(x, \xi_{0}\right)}-1\right|\left|\begin{array}{l}
\Psi_{0, i}^{t}\left(\lambda x, \lambda \xi_{0}\right)+e_{i}\left(t, \lambda x, \lambda \xi_{0}\right) \\
=\sup _{t \geq 0} \mid
\end{array}\right| \\
&= \sup _{t \geq 0}\left|\frac{\lambda \Psi_{0, i}^{t}\left(x, \xi_{0}\right)+e_{i}\left(t, \lambda x, \lambda \xi_{0}\right)}{\lambda \Psi_{0, i}^{t}\left(x, \xi_{0}\right)+\lambda e_{i}\left(t, x, \xi_{0}\right)}-1\right| \\
&= \sup _{t \geq 0}\left|\frac{-\lambda e_{i}\left(t, x, \xi_{0}\right)+e_{i}\left(t, \lambda x, \lambda \xi_{0}\right)}{\lambda \Psi_{0, i}^{t}\left(x, \xi_{0}\right)+\lambda e_{i}\left(t, x, \xi_{0}\right)}\right| \\
& \leq \sup _{t \geq 0}\left|\frac{-e_{i}\left(t, x, \xi_{0}\right)+\lambda^{-1} e_{i}\left(t, \lambda x, \lambda \xi_{0}\right)}{\eta}\right| \\
& \leq 2 \varepsilon \kappa\left(x, \xi_{0}\right) .
\end{aligned}
$$

Proof In this case $F_{0}(x, \xi)=\left(f_{0}^{T}(x, \phi(\xi)) g^{T}(\xi)\right)^{T}$, where $f_{0}$ is a kind of local approximation of $f$, i.e.

$$
\left[d_{x} \Phi_{1}^{s}\right]^{-1} f\left(\Phi_{1}^{s}(x), \phi\left(\Phi_{2}^{s}(\xi)\right)\right) \stackrel{s \rightarrow \pm \infty}{\longrightarrow} f_{0}(x, \phi(\xi))
$$

uniformly on compact sets. Since all conditions of Theorem 1 are satisfied for $F_{0}$, it has the pS property. Intuitively, in the domain of $\mathbb{R}^{n}$ where $F$ and $F_{0}$ are sufficiently close, the original system (2) has pAS property for a properly selected $\varepsilon>0$. Indeed, as before denote $\Psi$ and $\Psi_{0}$ the flows of $F$ and $F_{0}$ respectively, then $p_{2} \circ \Psi^{t}=p_{2} \circ \Psi_{0}^{t}$. Define $e(t)=p_{1}\left(\Psi^{t}\left(x, \xi_{0}\right)-\Psi_{0}^{t}\left(x, \xi_{0}\right)\right)$, then

$$
\begin{aligned}
\dot{e}(t)= & f_{0}\left(p_{1} \circ \Psi^{t}, u(t)\right)-f_{0}\left(p_{1} \circ \Psi_{0}^{t}, u(t)\right) \\
& +f\left(p_{1} \circ \Psi^{t}, u(t)\right)-f_{0}\left(p_{1} \circ \Psi^{t}, u(t)\right) .
\end{aligned}
$$

Finally, we apply the same steps than in Lemma 3 to conclude. 


\section{Discussion and application}

In this work scale invariance properties are studied. The system (2) admits pS property with respect to inputs from $\mathbb{U}$ if for any $\bar{u} \in \mathbb{R}^{m}$ and any $u \in \mathbb{U}$ their scaling by $\lambda>0$ leads to a proportional scaling of trajectories for all $t \geq 0$ :

$$
\frac{y(t, \sigma(\lambda \bar{u}), \lambda u)}{y(t, \sigma(\bar{u}), u)}=\beta(\lambda),
$$

where $\sigma: \mathbb{R}^{m} \rightarrow \mathbb{R}^{n}$ is the static characteristic of $(2)$ and $\beta: \mathbb{R}_{+}^{*} \rightarrow \mathbb{R}_{+}^{*}$ is some function (the case $\beta(s)=s^{p}$ for a $p \geq 0$ is mainly investigated here applying the homogeneity framework). The case with $\beta(\lambda)=1$ is called pFCD, it corresponds to a system giving a uniform response with respect to the input amplitude. This property can hold globally (i.e. for all $x \in \mathbb{R}^{n}$ ) and exactly, or locally (e.g., $|x| \leq X$ or $|x| \geq X$ for some $X>0$ ) and approximately:

$$
\sup _{t \geq 0}\left|\frac{y(t, \sigma(\lambda \bar{u}), \lambda u)}{y(t, \sigma(\bar{u}), u)}-\beta(\lambda)\right| \leq \varepsilon
$$

for some $\varepsilon>0$, then such a system is called (logarithmic) $\varepsilon$-pAS.

A procedure for applying the proposed theory for the scale invariance detection can be summarized as follows.

1. Consider a model described by the equations (2) with locally bounded inputs.

2. Verify Assumption 1. For that purpose it is necessary to select an Euler vector field $\nu_{1}$ and check homogeneity relations formulated for (2). The simplest candidate is $\nu_{1}(x)=\operatorname{diag}[\mathbf{r}] x$ with $\mathbf{r}=\left(r_{1}, \ldots, r_{n}\right)$, i.e. to check weighted homogeneity. In this case, Assumption 1 takes the following algebraic form:

$$
f\left(\Lambda_{\mathbf{r}} x, \lambda u\right)=\Lambda_{\mathbf{r}} f(x, u), \quad h\left(\Lambda_{\mathbf{r}} x\right)=\lambda^{p} h(x)
$$

for all $x \in \mathbb{R}^{n}$ and all $\lambda>0$ with some $p \geq 0$. If these relations are satisfied, then according to theorems 1 or 2 the system $(2)$ has $\mathrm{pS}(p>0)$ or $\mathrm{pFCD}$ $(p=0)$ property.

3. If Assumption 1 does not globally hold, then it can be checked locally looking for a pAS property. For this purpose the conditions of Corollary 2 should be satisfied. Take again $\nu_{1}(x)=\operatorname{diag}[\mathbf{r}] x$ with some $\mathbf{r}=\left(r_{1}, \ldots, r_{n}\right)$ (e.g., $\mathbf{r}=[1, \ldots 1])$ and assume that there exists $f_{0} \in \operatorname{Lip}\left(\mathbb{R}^{n+m}\right)$ such that

$$
\lim _{\lambda \rightarrow \lambda_{0}} \Lambda_{\mathbf{r}}^{-1} f\left(\Lambda_{\mathbf{r}} x, \lambda u\right)=f_{0}(x, u)
$$

uniformly on a sphere centered at the origin for some $\lambda_{0} \in\{0,+\infty\}$. If Jac $f_{0}(x, u(t)) \in C o\left(A_{1}, \ldots, A_{K}\right)$ for some $A_{k} \in \mathbb{R}^{n \times n}, 1 \leq k \leq K, K \in$ $\mathbb{N}^{*}$ and all these $A_{k}$ are asymptotically stable with a common Lyapunov function, then (2) is locally (close to the origin if $\lambda_{0}=0$ or far outside if $\left.\lambda_{0}=+\infty\right)$ pAS.

Let us show how this procedure can be applied for several models of biological cascades. 


\section{Validation on networked models}

In this section, we shall demonstrate how the scale invariance property can be verified using the homogeneity based results presented beforehand. We propose several examples, among which are academical ones and systems taken from the literature. As we will see, the methods developed in the preceding sections apply very easily and allow us to describe the behavior of the systems under interest.

One of the most restrictive conditions of the theory above deals with the inclusion of the $\operatorname{Jac} f_{0}$ or $\mathrm{Jac} F_{0}$ in a convex hull of matrices $A_{0}, \ldots, A_{K}$. The simplest and important case for verification of this condition is that approximations at zero or at infinity are linear systems. For example, let system (2) have the form

$$
\begin{aligned}
\dot{x}_{i}= & \sum_{j=1}^{n} a_{i, j} x_{j}+\sum_{l=1}^{n} c_{i, l} \frac{x_{l}}{1+\left|x_{l}\right|} \\
& +\sum_{v=1}^{n} d_{i, v} \frac{x_{v}^{2}}{1+\left|x_{v}\right|^{2}}+b_{i}^{T} u, 1 \leq i \leq n
\end{aligned}
$$

where $a_{i, j}, c_{i, l}$ and $d_{i, v}$ are real scalars, $b_{i} \in \mathbb{R}^{m}$. For $\mathbf{r}=[1, \ldots 1]$ the homogeneous approximation at zero is

$$
\dot{x}_{i}=\sum_{j=1}^{n}\left(a_{i, j}+c_{i, j}\right) x_{j}+b_{i}^{T} u, 1 \leq i \leq n,
$$

and homogeneous approximation at infinity is

$$
\dot{x}_{i}=\sum_{j=1}^{n} a_{i, j} x_{j}+b_{i}^{T} u, 1 \leq i \leq n,
$$

thus they both are linear systems and $\operatorname{Jac} f_{0}$ is a constant matrix. By the results above such a class of systems has to demonstrate pAS property close to the origin and far outside. There are many biological models that can be represented in this form, let us consider several examples.

Example 4 Recall the model of Repressilator [Elowitz \& Leibler, 2000] given in the introduction, it is in the form given above and for $\nu_{1}(x)=x \in \mathbb{R}^{6}$ its approximation at infinity for $i=1,2,3$ is

$$
\begin{aligned}
\dot{m}_{i}(t) & =u(t)-m_{i}(t), \\
\dot{p}_{i}(t) & =\beta\left(m_{i}(t)-p_{i}(t)\right) .
\end{aligned}
$$

Therefore, the system possesses the pAS property with $p=1$ (linear scaling) for big amplitudes of the state variables as it has been demonstrated by simulations in Fig. 1. 

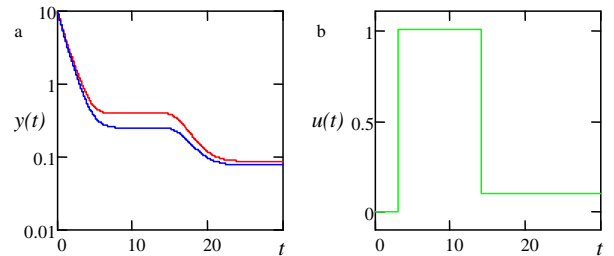

Fig. 2 The results of simulation for the system (7): (a) output trajectories $2 y_{u(t)}(t)$ and $y_{2 u(t)}(t)$ for the inputs $u(t)$ and $2 u(t)$; (b) input $u(t)$

Example 5 Consider a nonlinear cascade for some $n \in \mathbb{N}^{*}$ :

$$
\begin{aligned}
\dot{x}_{0} & =u-a_{0} x_{0}, \\
\dot{x}_{i} & =\frac{c_{i} x_{i-1}}{b_{i}+\left|x_{i-1}\right|}-a_{i} x_{i}, 1 \leq i \leq n, \\
y & =x_{n} .
\end{aligned}
$$

Let $\mathbb{U}$ be the class of piecewise constant inputs, then $g(\xi)=0, \phi(\xi)=\xi$ and $\nu_{2}(\xi)=\xi$. Denote $x=\left(x_{0}, x_{1}, \ldots, x_{n}\right)^{T}$ and take $\nu_{1}(x)=x$, then the system has the local approximation of degree 0 at 0

$$
\begin{aligned}
\dot{x}_{0} & =\xi-a_{0} x_{0}, \\
\dot{x}_{i} & =\frac{c_{i}}{b_{i}} x_{i-1}-a_{i} x_{i}, 1 \leq i \leq n, \\
\dot{\xi} & =0,
\end{aligned}
$$

and at $+\infty$

$$
\begin{aligned}
\dot{x}_{0} & =\xi-a_{0} x_{0}, \\
\dot{x}_{i} & =-a_{i} x_{i}, 1 \leq i \leq n, \\
\dot{\xi} & =0,
\end{aligned}
$$

which are stable linear systems (their stability follows the lower triangular structure of both). Thus the system (7) should demonstrate pAS property in almost all state space $\mathbb{R}^{n+1}$ for $p=1$ (linear scaling). The results of the system simulation for

$$
\begin{gathered}
n=2, a_{0}=a_{1}=a_{2}=1, c_{1}=c_{2}=1, \\
b_{1}=1, b_{2}=2,
\end{gathered}
$$

are shown in Fig. 2. The piecewise constant input $u(t)$ is presented in Fig. 2,b. The output trajectories $2 y_{u(t)}(t)$ and $y_{2 u(t)}(t)$ corresponding to the inputs $u(t)$ and $2 u(t)$, respectively, are plotted in the logarithmic scale in Fig. 2,a.

Example 6 Following [Ropers et al., 2009, Smolen et al., 2000], consider a model of genetic regulatory network with a positive feedback:

$$
\begin{aligned}
& \dot{x}_{1}=u(t)+\kappa_{1} \frac{x_{2}^{n}}{\theta_{1}+x_{2}^{n}}-\gamma_{1} x_{1}, \\
& \dot{x}_{2}=\kappa_{2} x_{1}-\gamma_{2} x_{2}, y=x_{2},
\end{aligned}
$$



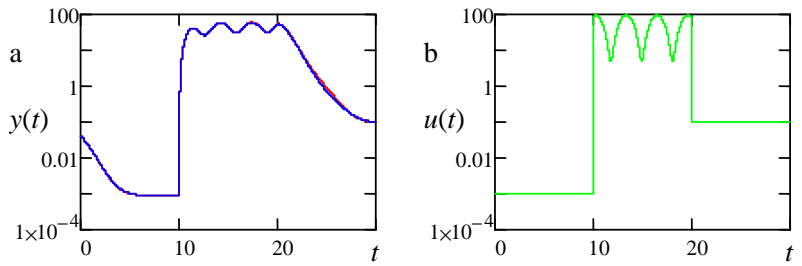

Fig. 3 The results of simulation for the system (8): (a) output trajectories $2 y_{u(t)}(t)$ and $y_{2 u(t)}(t)$ for the inputs $u(t)$ and $2 u(t) ;(\mathrm{b})$ input $u(t)$

where $x_{1} \in \mathbb{R}_{+}$and $x_{2} \in \mathbb{R}_{+}$are mRNA and protein concentrations respectively, $\kappa_{i}>0, \gamma_{i}>0$ are production and degradation rate constants respectively, $\theta_{1}>0, n>0$ are constant parameters, $u(t) \in \mathbb{R}_{+}$is external input modeled by a biased sinusoid of the frequency $\omega$. Take $\nu_{1}(x)=x$ and $\nu_{2}(\xi)=\xi$, then the system has the same local approximation of degree 0 at 0 and at $+\infty$

$$
\begin{aligned}
& \dot{x}_{1}=\xi_{1}+\xi_{2}-\gamma_{1} x_{1}, \\
& \dot{x}_{2}=\kappa_{2} x_{1}-\gamma_{2} x_{2}, \\
& \dot{\xi_{1}}=0, \\
& \dot{\xi}_{2}=\xi_{3}, \dot{\xi}_{3}=-\omega^{2} \xi_{2},
\end{aligned}
$$

which is a stable linear system (again, due to its lower triangular structure). Thus, the system (8) should demonstrate pAS property in almost all state space $\mathbb{R}^{n+1}$ for $p=1$ (i.e. the trajectories of the system are scaled proportionally the input amplitude). In other words, in (8) the shape of stimulus $u$ defines time behavior of concentrations, and the amplitude of stimulus does not influence the shape but only amplitude of concentration transients.

The results of the system simulation for

$$
n=2, \kappa_{1}=\kappa_{2}=\theta_{1}=1, \gamma_{1}=\gamma_{2}=1.5, \omega=2
$$

are shown in Fig. 3. The piecewise continuous input $u(t)$ is presented in Fig. 3,b in logarithmic scale. The output trajectories $2 y_{u}(t)$ and $y_{2 u}(t)$ corresponding to the inputs $u(t)$ and $2 u(t)$, respectively, are plotted in logarithmic scale in Fig. 3,a.

Example 7 Finally consider the model of Goodwin oscillator from [Gonze \& Abou-Jaoudé, 2013] (example of a biochemical oscillator with negative feedback):

$$
\begin{aligned}
& \dot{x}_{1}=u+v_{1} \frac{K^{c}}{K^{c}+x_{3}^{c}}-k_{1} x_{1}, \\
& \dot{x}_{2}=v_{2} x_{1}-k_{2} x_{2}, \\
& \dot{x}_{3}=v_{3} x_{2}-k_{3} x_{3},
\end{aligned}
$$

where $x_{1}, x_{2}$ and $x_{3}$ are the concentrations of mRNA, protein, and end product, respectively; $v_{1}, v_{2}$ and $v_{3}$ determine the rates of transcription, translation, and catalysis; $k_{i}, i=1,2,3$ are the rate constants for degradation of 

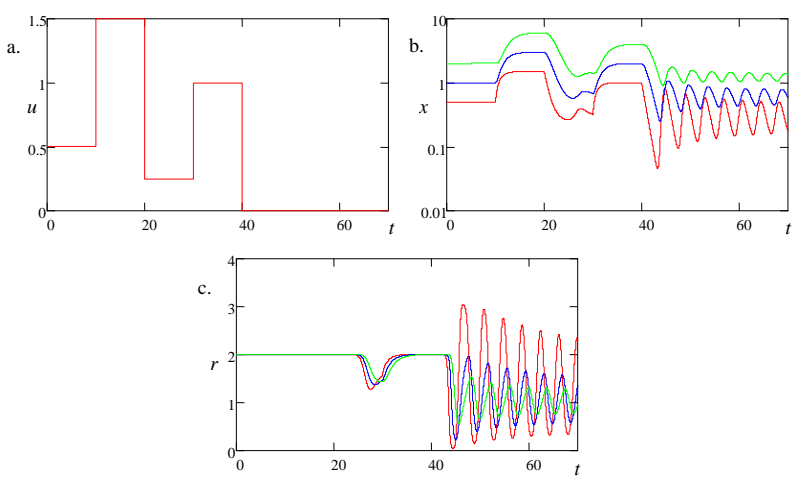

Fig. 4 The results of simulation for the Goodwin oscillator: (a) input $u(t)$; (b) the state variables $x_{i}(t), i=1,2,3$ for the input $u(t)$; (c) the ratio between state variables for $2 u(t)$ and $u(t)$

each component; $1 / K$ is the binding constant of end product to transcription factor; $c>8$ is a measure of the cooperativity of end product repression; $u$ is the external stimulation input, which is assumed piecewise constant. For properly selected parameters, this model demonstrates oscillations for $u=0$. For $\nu_{1}(x)=x$ and $\nu_{2}(\xi)=\xi$ this model has a linear approximation at infinity:

$$
\begin{aligned}
\dot{x}_{1} & =\xi-k_{1} x_{1}, \\
\dot{x}_{2} & =v_{2} x_{1}-k_{2} x_{2}, \\
\dot{x}_{3} & =v_{3} x_{2}-k_{3} x_{3}, \\
\dot{\xi} & =0
\end{aligned}
$$

that is stable. Therefore, the Goodwin oscillator has to demonstrate pAS for large magnitudes of the state vector. The results of simulation for

$$
K=1 ; k_{i}=1, v_{i}=2 \quad i=1,2,3 ; c=10
$$

are shown in Fig 4. The input is shown in Fig 4,a and the corresponding state trajectory of the oscillator in Fig 4,b (in the logarithmic scale). Therefore, for $u \neq 0$ the system converges to a steady-state, and when $u=0$ an oscillatory mode is initiated. In Fig $4, \mathrm{c}$ the ratios $r_{i}=\frac{x_{i}(t, \sigma(\lambda \bar{u}), \lambda u)}{x_{i}(t, \sigma(\bar{u}), u)}$ are presented for $i=1,2,3$ and $\lambda=2$. If the amplitude of $x$ stay large, then these $r_{i}$ are close to $\lambda$ demonstrating a nice logarithmic pAS. The ratios start oscillating at the end, when $|x|$ becomes small and the trajectory enters in oscillatory motion. The observation of the scaling invariance for Goodwin oscillator seems an interesting demonstration of the proposed theory. 
Example 8 Let us consider the model of circadian rhythms in Neurospora from [Leloup et al., 1999]:

$$
\begin{aligned}
\dot{M}(t) & =v_{s}(t) \frac{K_{I}^{n}}{K_{I}^{n}+F_{N}^{n}(t)}-v_{m} \frac{M(t)}{K_{m}+M(t)} \\
\dot{F}_{C}(t) & =k_{m} M(t)-v_{d} \frac{F_{C}(t)}{K_{d}+F_{C}(t)}-k_{1} F_{C}(t)+k_{2} F_{N}(t), \\
\dot{F}_{N}(t) & =k_{1} F_{C}(t)-k_{2} F_{N}(t),
\end{aligned}
$$

where $M, F_{C}$, and $F_{N}$ denote, respectively, the concentrations of the frq mRNA and of the cytosolic and nuclear forms of FRQ. The input $v_{s}$ denotes the rate of $f r q$ transcription increasing in the light phase. A description of all the other parameters of the model can be found in [Leloup et al., 1999]. Take $\nu_{1}(x)=x$ for $x=\left[\begin{array}{lll}M & F_{C} & F_{N}\end{array}\right]^{T}$, then the system has a local approximation of degree 0 at $+\infty$ givenby

$$
\begin{aligned}
& \dot{M}(t)=v_{s}(t)-\frac{v_{m}}{K_{m}} M(t) \\
& \dot{F}_{C}(t)=k_{m} M(t)-\left[k_{1}+\frac{v_{d}}{K_{d}}\right] F_{C}(t)+k_{2} F_{N}(t), \\
& \dot{F}_{N}(t)=k_{1} F_{C}(t)-k_{2} F_{N}(t),
\end{aligned}
$$

which is stable. Thus, the model of circadian rhythms in Neurospora admits the pAS property for small values of concentrations with $p=1$. The results of the system simulation for

$$
\begin{gathered}
v_{s}(t)=\left\{\begin{array}{ll}
1.6 & \text { if } t \in[72,120] \\
0.016 & \text { otherwise }
\end{array} ;\right. \\
v_{m}=0.505, v_{d}=1.4, k_{m}=k_{1}=0.5, k_{2}=0.6, \\
K_{I}=1, K_{m}=0.5, K_{d}=0.13, n=4 ; \\
{\left[M(0) F_{C}(0) F_{N}(0)\right]=[0.159] \times 10^{-3}}
\end{gathered}
$$

are shown in Fig. 5. The corresponding behavior of concentrations is given in Fig. 5, a, and the ratios $r_{i}=\frac{x_{i}(t, \sigma(\lambda \bar{u}), \lambda u)}{x_{i}(t, \sigma(\bar{u}), u)}$ are presented for $i=1,2,3$ and for the scaling $\lambda=3$ in Fig. 5,b. If the values of concentrations become small, then all ratios $r_{i}$ are approaching $\lambda$, which is, by definition, an illustration of scale invariance.

Similarly the model of circadian rhythms in Drosophila from [Leloup et al., 1999] can be analyzed.

Therefore, these results of simulation confirm the theoretical developments of the paper.

Let us stress that scale invariance is important in the design of a model because it represents an important feature of real systems, explaining the influence of the input, and therefore has to be represented in the corresponding model. Conversely, if it was recognized that a model is scale invariant, then 

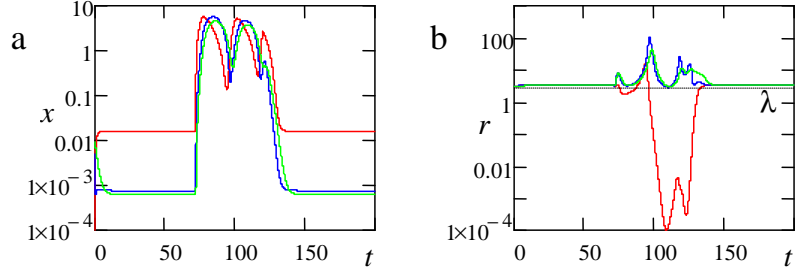

Fig. 5 The results of simulation for Neurospora: (a) the model trajectory; (b) the ratios between variables from the part (a) of the figure and with variables obtained for initial conditions and input scaled by $\lambda$

it should be verified whether the real system presents an invariance property. Consequently, during the modeling process of any system, the consistency between the scale invariance of the real system and of the model has to be carefully watched. Taking into account the models presented in the paper, e.g. Repressilator, Goodwin oscillator, circadian rhythms in Neurospora, where the presence of scale invariance has been detected in this work, the presence of this phenomenon should be analyzed or understood in the real systems.

\section{Conclusion}

First, the problem of scalability of trajectories for dynamical systems has been studied. The system has the property of scalability of trajectories if a scaling of the input and initial conditions leads to a proportional scaling of the whole trajectory. Such a behavior is important for many biological systems, which should demonstrate almost the same output response independently in external stimulus, a property called fold change detection, which is a particular variant of scalability. Another example is the Weber-Fechner law, which describes a rule of human perception (to be detected, the response has to be proportional to the stimulus). The conditions of scalability are important for understanding qualitative properties of biological systems. In this work these conditions have been obtained using the homogeneity framework. The advantage is that the local or global homogeneity of a vector field can be checked doing a simple calculus. Therefore, in practice, the verification of this property is rather straightforward. In this work the relations between (approximate) scalability and (local) homogeneity are established.

Second, the present work contains some mathematical developments. Conditions on closeness of solutions of the original system and its local approximation are given. In the theory of homogeneity, it is well known that a system inherits stability/instability properties from its homogeneous approximation. The main result of this work claims that under mild additional conditions the solutions of the system and its homogeneous approximation stay close in the corresponding domains.

The theoretical results are confirmed by numerical experiments for models of biological cascades. 
This development also opens several new directions of research, in particular, what happens with homogeneous approximations and their stability in cascades, and is it possible from the scale invariance of subsystems to make a conclusion of the inheritance of this property by the cascade? Another direction deals with the quantitative evaluation of the domain in the state space where the pAS property is satisfied and with which error. All these questions shall be addressed in future works.

This theoretical development can also be used for design of models having fold change detection in cascades. For instance, consider the following cascade:

$$
\begin{aligned}
& \dot{x}_{1}=f\left(x_{1}, u\right), y_{1}=h\left(x_{1}\right) ; \\
& \dot{x}_{2}=\frac{y_{1}}{u^{p}}-a x_{2}, y_{2}=x_{2},
\end{aligned}
$$

where $x_{1} \in \mathbb{R}^{n}$ and $y_{1} \in \mathbb{R}$ are the state and output of the first system that is $\mathrm{pS}$ (or pAS for small or big values of the state $x_{1}$ ) with respect to inputs $u$ from $\mathbb{U}$ with $\beta(\lambda)=\lambda^{p}$ for some $p \geq 0$; while $x_{2} \in \mathbb{R}$ and $y_{2} \in \mathbb{R}$ are the state and output of the second system in cascade and $a>0$ is a parameter (for $p=1$ this system is similar to considered in [Shoval et al., 2011, Skataric \& Sontag, 2012, Hamadeh et al., 2013]). Then the cascade has pFCD property globally (or locally for small or big values of the state $x_{1}$ ).

\section{References}

[Alon, 2006] Alon, U. (2006). An Introduction to Systems Biology: Design Principles of Biological Circuits. Chapman \& Hall.

[Andrieu et al., 2008] Andrieu, V., Praly, L., \& Astolfi, A. (2008). SIAM J. Control Optimization, 47 (4), 1814-1850.

[Bacciotti \& Rosier, 2005] Bacciotti, A. \& Rosier, L. (2005). Lyapunov Functions and Stability in Control Theory. Springer, 2nd edition.

[Bernuau et al., 2014] Bernuau, E., Efimov, D., \& Perruquetti, W. (2014). In: Proc. of ECC 2014 ,

[Bernuau et al., 2013a] Bernuau, E., Polyakov, A., Efimov, D., \& Perruquetti, W. (2013a). In: Proc. of ECC 2013 ,

[Bernuau et al., 2013b] Bernuau, E., Polyakov, A., Efimov, D., \& Perruquetti, W. (2013b). Systems \& Control Letters, 62 (12), 1159-1167.

[Efimov \& Perruquetti, 2010] Efimov, D. \& Perruquetti, W. (2010). In: Proc. IFAC NOLCOS Symp. pp. 1379-1384,.

[Elowitz \& Leibler, 2000] Elowitz, M. B. \& Leibler, S. (2000). Nature, 403, 335-338.

[Gisiger, 2001] Gisiger, T. (2001). Biological Reviews of the Cambridge Philosophical Society, 76 (02), 161-209.

[Gonze \& Abou-Jaoudé, 2013] Gonze, D. \& Abou-Jaoudé, W. (2013). PLoS ONE, 8 (8), e69573.

[Hamadeh et al., 2013] Hamadeh, A., Ingalls, B., \& Sontag, E. (2013). Proc. Royal Society Interface, 10, 20120935.

[Hermes, 1986] Hermes, H. (1986). SIAM J. Control Optim. 24, 731.

[Ingalls \& Sontag, 2002] Ingalls, B. \& Sontag, E. (2002). J. Franklin Inst. 339, 211-229.

[Kawski, 1995] Kawski, M. (1995). In: Proc. IFAC Nonlinear Control Symposium, (Krener, A. \& Mayne, D., eds) pp. 164-169, Lake Tahoe, CA:.

[Khomenuk, 1961] Khomenuk, V. V. (1961). Izvestia vuzov. Mathematica. 3(22), 157-164. (in Russian). 
[Leloup et al., 1999] Leloup, J.-C., Gonze, D., \& Goldbeter, A. (1999). Journal of Biological Rhythms, 14 (6), 433-448.

[Pavlov et al., 2004] Pavlov, A., Pogromsky, A., van de Wouw, N., \& Nijmeijer, H. (2004). Systems \& Control Letters, 52, 257-261.

[Ropers et al., 2009] Ropers, D., de Jong, H., \& Geiselmann, J. (2009). Systems Biology and Synthetic Biology chapter Mathematical modeling of genetic regulatory networks: Stress responses in Escherichia coli, pp. 235-271. Hoboken, NJ: John Wiley \& Sons.

[Rosier, 1992] Rosier, L. (1992). Systems \& Control Letters, , 19, 467-473.

[Shoval et al., 2011] Shoval, O., Alon, U., \& Sontag, E. (2011). SIAM Journal on Applied Dynamical Systems, , 10, 857-886.

[Skataric \& Sontag, 2012] Skataric, M. \& Sontag, E. (2012). PLoS Computational Biology, 8, e1002748.

[Smolen et al., 2000] Smolen, P., Baxter, D., \& Byrne, J. (2000). Bulletin of Mathematical Biology, , 62 (2), 247-292.

[Zubov, 1958] Zubov, V. (1958). Izvestia vuzov. Mathematica., 1, 80-88. in Russian. 